\title{
Longitudinal Assessment of Cerebral $\beta$-Amyloid Deposition in Mice Overexpressing Swedish Mutant $\beta$-Amyloid Precursor Protein Using ${ }^{18}$ F-Florbetaben PET
}

\author{
Axel Rominger*1, Matthias Brendel*1, Steffen Burgold ${ }^{2}$, Kevin Keppler ${ }^{2}$, Karlheinz Baumann ${ }^{3}$, Guoming Xiong ${ }^{1}$, \\ Erik Mille $^{1}$, Franz-Josef Gildehaus ${ }^{1}$, Janette Carlsen ${ }^{1}$, Juli Schlichtiger ${ }^{1}$, Sabrina Niedermoser ${ }^{1}$, Björn Wängler ${ }^{1}$, \\ Paul Cumming ${ }^{4}$, Harald Steiner ${ }^{5,6}$, Jochen Herms ${ }^{2}$, Christian Haass ${ }^{5-7}$, and Peter Bartenstein ${ }^{1,7}$ \\ ${ }^{1}$ Department of Nuclear Medicine, Ludwig-Maximilians-University of Munich, Munich, Germany; ${ }^{2}$ Department of Translational \\ Brain Research, German Center for Neurodegenerative Diseases (DZNE) Munich, Ludwig-Maximilians-University of Munich, \\ Munich, Germany; ${ }^{3}$ F. Hoffmann-La Roche AG, pRED, Pharma Research and Early Development, DTA CNS, Basel, Switzerland; \\ ${ }^{4}$ ABX, Radeberg, Germany; ${ }^{5}$ Adolf-Butenandt-Institute, Biochemistry, Ludwig-Maximilians-University of Munich, Munich, Germany; \\ ${ }^{6}$ German Center for Neurodegenerative Diseases (DZNE), Munich, Germany; and ${ }^{7}$ Munich Cluster for Systems Neurology (SyNergy), \\ Munich, Germany
}

The progression of $\beta$-amyloid deposition in the brains of mice overexpressing Swedish mutant $\beta$-amyloid precursor protein (APPSwe), a model of Alzheimer disease (AD), was investigated in a longitudinal PET study using the novel $\beta$-amyloid tracer ${ }^{18} \mathrm{~F}$ florbetaben. Methods: Groups of APP-Swe and age-matched wild-type (WT) mice (age range, 10-20 mo) were investigated. Dynamic emission recordings were acquired with a small-animal PET scanner during 90 min after the administration of ${ }^{18} \mathrm{~F}$-florbetaben (9 MBq, intravenously). After spatial normalization of individual PET recordings to common coordinates for mouse brain, binding potentials $\left(\mathrm{BP}_{\mathrm{ND}}\right)$ and standardized uptake value ratios (SUVRs) were calculated relative to the cerebellum. Voxelwise analyses were performed using statistical parametric mapping (SPM). Histochemical analyses and ex vivo autoradiography were ultimately performed in a subset of animals as a gold standard assessment of $\beta$-amyloid plaque load. Results: SUVRs calculated from static recordings during the interval of 30-60 min after tracer injection correlated highly with estimates of $\mathrm{BP}_{\mathrm{ND}}$ based on the entire dynamic emission recordings. ${ }^{18} \mathrm{~F}$-florbetaben binding did not significantly differ in APP-Swe mice and WT animals at 10 and 13 mo of age. At 16 mo of age, the APP-Swe mice had a significant $7.9 \%$ increase $(P<0.01)$ in cortical ${ }^{18} \mathrm{~F}$-florbetaben uptake above baseline and at 20 mo there was a $16.6 \%$ increase $(P<0.001)$, whereas WT mice did not show any temporal changes in tracer uptake during the interval of follow-up. Voxelwise SPM analyses revealed the first signs of increased cortical binding at 13 mo and confirmed progressive binding increases in both the frontal and the temporal cortices $(P<0.001$ uncorrected) to $20 \mathrm{mo}$. The SUVR strongly correlated with percentage plaque load $(R=0.95, P<0.001)$. Conclusion: In the first longitudinal PET study in an AD mouse model using the novel $\beta$-amyloid tracer ${ }^{18} \mathrm{~F}$-florbetaben, the temporal and spatial progression of amyloidogenesis in the brain of APP-Swe mice were sensitively monitored. This method should afford the means for preclinical testing of novel therapeutic approaches to the treatment of AD.

Received Sep. 24, 2012; revision accepted Jan. 23, 2013.

For correspondence or reprints contact: Axel Rominger, Department of Nuclear Medicine, University of Munich, Marchioninistrasse 15, 81377 Munich, Germany.

E-mail: axel.rominger@med.uni-muenchen.de

${ }^{*}$ Contributed equally to this work.

Published online May 31, 2013.

COPYRIGHT (c) 2013 by the Society of Nuclear Medicine and Molecular Imaging, Inc.
Key Words: Alzheimer disease; $\beta$-amyloid plaque load; small-animal PET; transgenic mouse model; ${ }^{18} \mathrm{~F}$-florbetaben

J Nucl Med 2013; 54:1127-1134

DOI: 10.2967/jnumed.112.114660

A

lzheimer disease (AD) is the most common form of dementia, with exponentially increasing incidence as a function of age among the elderly, which is imposing an onerous burden on health care in societies with aging populations (1). There is an urgent need to find biomarkers to predict future clinical decline and likewise for use as outcome measures in clinical trials of innovative disease-modifying agents (2). The main neuropathologic features of $\mathrm{AD}$, which include the accumulation of extracellular $\beta$-amyloid plaques and intracellular neurofibrillary tangles (3), are emulated in transgenic animal models for $\mathrm{AD}(4,5)$. Although current transgenic AD models obviously do not manifest all clinical and pathologic aspects of AD (6), they are nonetheless of heuristic value for the testing of interventions that address certain aspects of the disease, notably the formation of $\beta$-amyloid plaques. In this regard, molecular imaging of $\beta$-amyloid by PET has emerged as an important endpoint for assessing the extent of disease progression, although only limited imaging data are available in rodent AD models (7-11). The $\beta$-amyloid ligand ${ }^{18} \mathrm{~F}$-florbetaben, with high affinity for fibrillary $\beta$-amyloid in vitro, is currently in clinical development by Bayer Healthcare and Piramal Imaging (12-14) and has proven to have high sensitivity and specificity in clinical PET examinations for distinguishing $\mathrm{AD}$ patients from healthy control subjects (15). However, the applicability of this agent for monitoring in longitudinal studies the accumulation of fibrillary $\beta$-amyloid in $\mathrm{AD}$ mice remains to be established.

The aim of this longitudinal PET study was to investigate the novel amyloid tracer ${ }^{18} \mathrm{~F}$-florbetaben in the transgenic APP-Swe mouse model, which expresses the Swedish double-mutation of the amyloid precursor protein (APP). We performed sequential ${ }^{18} \mathrm{~F}$ florbetaben PET examinations in groups of transgenic and wildtype (WT) mice (age range, 10-20 mo) and examined different approaches to quantitation of the $\beta$-amyloid signal in the brain. In 


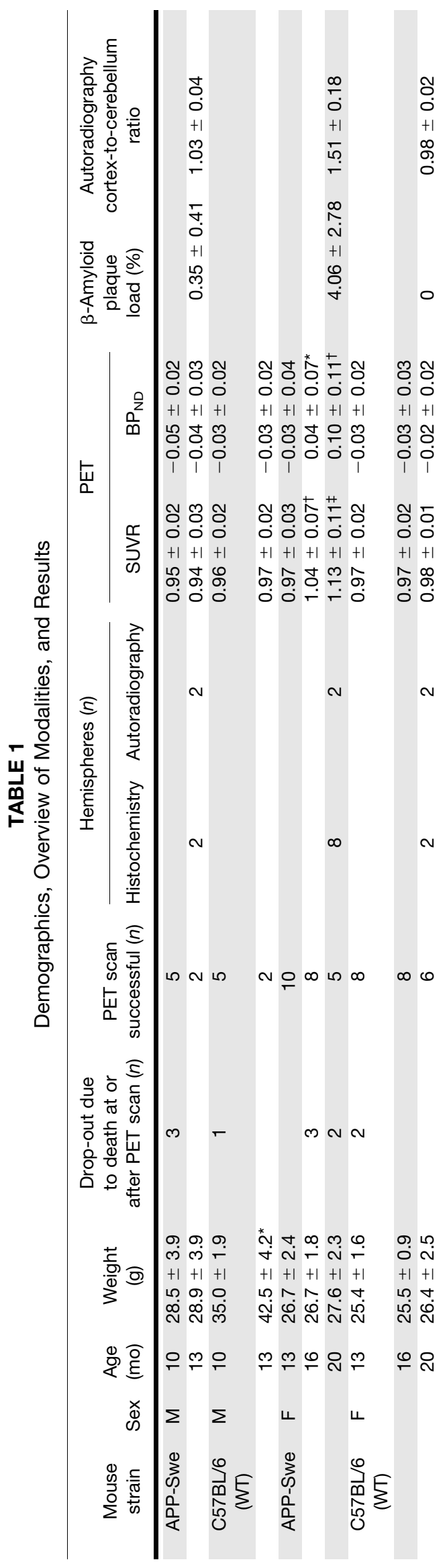

addition, the PET results were validated in a subset of animals relative to findings by autoradiography ex vivo and histochemistry.

\section{MARTERIALS AND METHODS}

\section{Radiochemistry}

The ${ }^{18} \mathrm{~F}$-florbetaben precursor provided by Bayer Pharma AG was radiolabeled via the method of Zhang et al. (12), with slight modifications. The detailed description of this radiosynthesis is presented in the supplemental data (supplemental materials are available online only at http://jnm.snmjournals.org). This procedure yields a radiochemical purity exceeding $98 \%$ and specific activity of $80 \mathrm{GBq} / \mu \mathrm{mol}$ at the end of synthesis.

\section{Animals}

All experiments were performed in compliance with the National Guidelines for Animal Protection, Germany, and the approval of the animal care committee. Transgenic mice overexpressing human APP with the Swedish double mutation (K670N, M671L) driven by the mouse Thy 1.2 promoter were generated as described earlier (16). These APP-Swe mice were backcrossed to C57BL/6 mice to generate a line with less than $5 \%$ DBA2 background (as determined by microsatellites genotyping). Mice heterozygous for the transgene start to accumulate $\beta$-amyloid at the age of approximately 9 mo and develop $\beta$-amyloid plaques around the age of $12 \mathrm{mo}$, mainly in the cortical mantle. At 18 mo of age, numerous amyloid plaques positively stained with Congo red are observed through the brains of these animals (Karlheinz Baumann, personal communication).

In the pilot study, 5 male heterozygous APP-Swe mice from Hoffmann-La Roche and 5 male C57BL/6 control mice purchased from Charles River Laboratories were investigated by molecular imaging. For the main study, 10 female heterozygous APP-Swe mice and 10 female age-matched C57BL/6 mice were used. Animals were housed in a temperature- and humidity-controlled environment with a 12-h light-dark cycle, with free access to food (Ssniff) and water.

\section{Study Design}

The study design entailed a longitudinal series of PET sessions at different ages. A detailed overview of the design is provided in Table 1.

Pilot Study Phase. PET recordings were obtained in 5 male transgenic and 5 male WT mice at the age of 10 and $13 \mathrm{mo}$; in 2 of these animals, autoradiography ex vivo and histochemistry were performed immediately after the final PET session

Main Study Phase. PET recordings were obtained in 10 female transgenic and 10 female WT mice at the age of 13, 16, and 20 mo, in conjunction with ex vivo autoradiography in 2 transgenic and 2 WT animals at 20 mo of age. Histochemistry was performed in all surviving transgenic animals at 20 mo.

\section{PET Recordings: Data Acquisition}

Mice were anesthetized with isoflurane (1.5\%, delivered at 3.5 $\mathrm{L} / \mathrm{min}$ ) and placed in the aperture of the Siemens Inveon DPET as described previously (17). On injection of $8.9 \pm 2.7 \mathrm{MBq}$ of ${ }^{18} \mathrm{~F}-$ florbetaben in $150 \mu \mathrm{L}$ of saline to a tail vein, a 90-min dynamic emission recording was initiated, followed by a 15 -min transmission scan using a rotating ${ }^{57} \mathrm{Co}$ point source. The dynamic acquisition protocol consisted of 19 frames $(3 \times 10,1 \times 30,4 \times 60,5 \times$ 300 , and $6 \times 600 \mathrm{~s}$ ). After recovery from anesthesia, mice were returned to their home cages. In the case of terminal experiments, 
animals were killed by cervical dislocation while still deeply anesthetized, before rapid removal of the brain. In the pilot study, an additional dynamic PET recording with ${ }^{18}$ F-FDG (11.4 \pm 0.2 $\mathrm{MBq}$ ) lasting $15 \mathrm{~min}$ was obtained after the completion of the ${ }^{18} \mathrm{~F}$-florbetaben scan, without the mouse being removed from the scanner. The reconstructed ${ }^{18} \mathrm{~F}-\mathrm{FDG}$ emission image was used to facilitate a precise manual coregistration of ${ }^{18} \mathrm{~F}$-florbetaben scans in the pilot study, but this measure was otherwise deemed uninformative for the main study.

\section{Image Reconstruction and PET Data Analyses}

Reconstruction was performed with 3-dimensional orderedsubset expectation maximization and 3-dimensional maximum a posteriori (4 and 32 iterations, respectively); a zoom factor of 1.0; and scatter, attenuation, and decay correction, resulting in a final voxel dimension of $0.78 \times 0.78 \times 0.8 \mathrm{~mm}$. A ${ }^{18} \mathrm{~F}$ florbetaben template was generated by coregistration of the ${ }^{18} \mathrm{~F}$-FDG and ${ }^{18} \mathrm{~F}$-florbetaben summation images to a mouse brain MR imaging atlas (18) using the PMOD fusion tool (version 3.302; PMOD Technologies Ltd.). A total-body volume of interest (VOI) was drawn to quantify the injected dose. Both VOIand voxel-based analyses were performed after manual rigid-body coregistration and accurate alignment by manual reregistration of individual ${ }^{18} \mathrm{~F}$-florbetaben summation images on the ${ }^{18} \mathrm{~F}$-florbetaben template. For the purpose of quality control of the PET/MR image-fusion process, longitudinal PET data of individual mice were checked for exact overlapping so as to guarantee the best possible intraindividual coregistrations.

A cerebellar VOI comprising $36 \mathrm{~mm}^{3}$, a frontal cortex VOI comprising $19 \mathrm{~mm}^{3}$, and a hippocampal VOI comprising $9 \mathrm{~mm}^{3}$ were designed so as to minimize signal loss due to partial-volume effects (Fig. 1) $(11,19)$. The binding potential $\left(\mathrm{BP}_{\mathrm{ND}}\right)$ was calculated voxelwise in the cortical VOI from the entire dynamic recordings using a linear graphic method (20), with the cerebellum serving as the reference tissue. The ${ }^{18} \mathrm{~F}$-florbetaben standardized uptake value ratio (SUVR) was calculated voxelwise and in the cortical VOI using different static time frames between 20 and 90 min after injection. For whole-brain voxelwise comparisons between groups of transgenic and WT mice, statistical parametric mapping (SPM) was performed using SPM5 routines (Wellcome Department of Cognitive Neurology) implemented in MATLAB (version 7.1; MathWorks Inc.), adapted from Sawiak et al. (21) for mouse data.

\section{Ex Vivo Autoradiography}

Autoradiography ex vivo was performed immediately after the ${ }^{18}$ F-florbetaben PET scanning. A detailed description of the procedure is provided in the supplemental data. Resulting images were analyzed with AIDA image-analyzing software (version 4.50; Raytest $\mathrm{GmbH}$ ). Each value was normalized with respect to the cerebellum as the control brain region (ratio to cerebellum).

\section{Histochemistry: Acquisition and Image Analysis}

A detailed description of the histochemistry acquisition procedure is provided in the supplemental data. For image analysis, we defined a region corresponding to the frontal cortex VOI from PET image analysis. The 3-dimensional image stacks were maximum-intensity-projected. The area and number of plaques were automatically counted in segmented images using Imaris software (Imaris 7.4.2; Bitplane). The corresponding volume for each plaque was calculated from the plaque area, assuming spheric plaques. Plaque load was calculated by integrating the volume of all

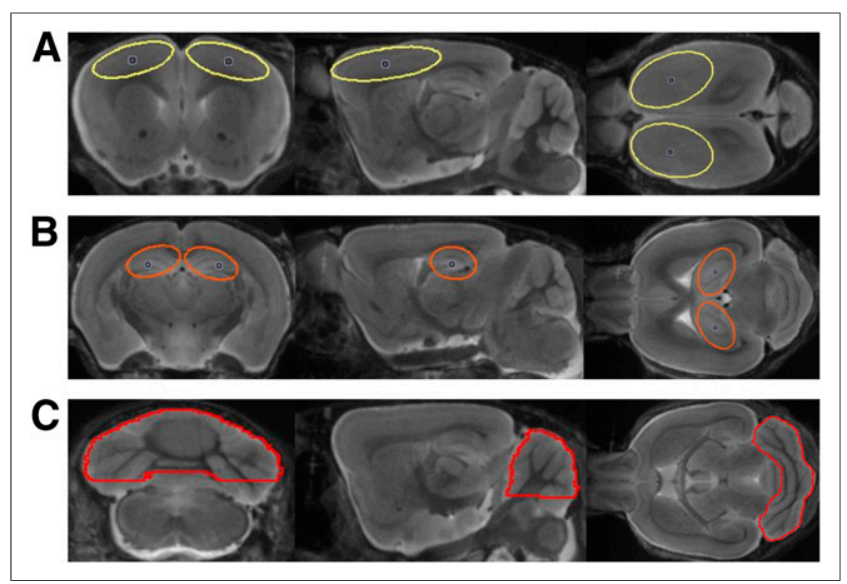

FIGURE 1. Definitions of cortical (yellow) (A), hippocampal (orange) (B), and cerebellar (red) (C) VOls in mouse brain MR imaging atlas (18) in coronal, sagittal, and axial slices.

plaques and relating it to the imaged volume. All experiments were performed in a manner blind to the results of PET analysis.

\section{Statistics}

Group comparisons of VOI-based PET results in transgenic versus WT mice were performed with the 2-sample Student $t$ test using IBM SPSS Statistics (version 20.0; SPSS). For correlation analyses, Pearson coefficients of correlation $(R)$ were calculated. A threshold of $P$ less than 0.05 was considered to be significant for rejection of the null hypothesis. For the voxelwise group comparison (SPM5) of transgenic versus WT mice, 2-sample $t$ tests were performed, with a significance threshold of $P$ less than 0.001 , uncorrected for multiple comparisons.

\section{RESULTS}

\section{Animals}

During the pilot and main studies, 58 PET scans were acquired in up to 3 sessions per animal. The drop-out rate was $20.1 \%$ per/ after scan session, mainly because of the high sensitivity of the transgenic animals to prolonged intervals of anesthesia. No significant sex differences were observed with regard to quantitation; therefore, pilot and main study results at 13 mo were combined. The weight of male WT animals in the pilot study increased with age, whereas the weight for all female animals remained constant over time. All details of animal characteristics are displayed in Table 1.

\section{Methodology and VOI-Based Analyses}

Mean $( \pm S D)$ target-to-cerebellum ratios in transgenic mice aged 13 and $20 \mathrm{mo}$ and for pooled WT mice aged 10-20 mo, along with corresponding petrous bone-to-cerebellum ratios, are shown in Figure 2A. For illustrative purposes of extracerebral activity, serial PET recordings from different time frames are depicted in Figure 2B, and a cerebellum reference tissue Logan plot for the cortical VOI in 20-mo-old transgenic animals is presented in Figure $2 \mathrm{C}$. The time-activity curves suggest the attainment of equilibrium binding in the brain by about $20 \mathrm{~min}$ in all groups. The highest correlation of $\mathrm{BP}_{\mathrm{ND}}$, calculated for the entire 90-min recording, and static SUVR $(R=0.98, P<0.0001)$ was found for the time frame of 30-60 min after injection (Fig. 3), revealing this time window to be best suitable for further static analyses. Furthermore, 

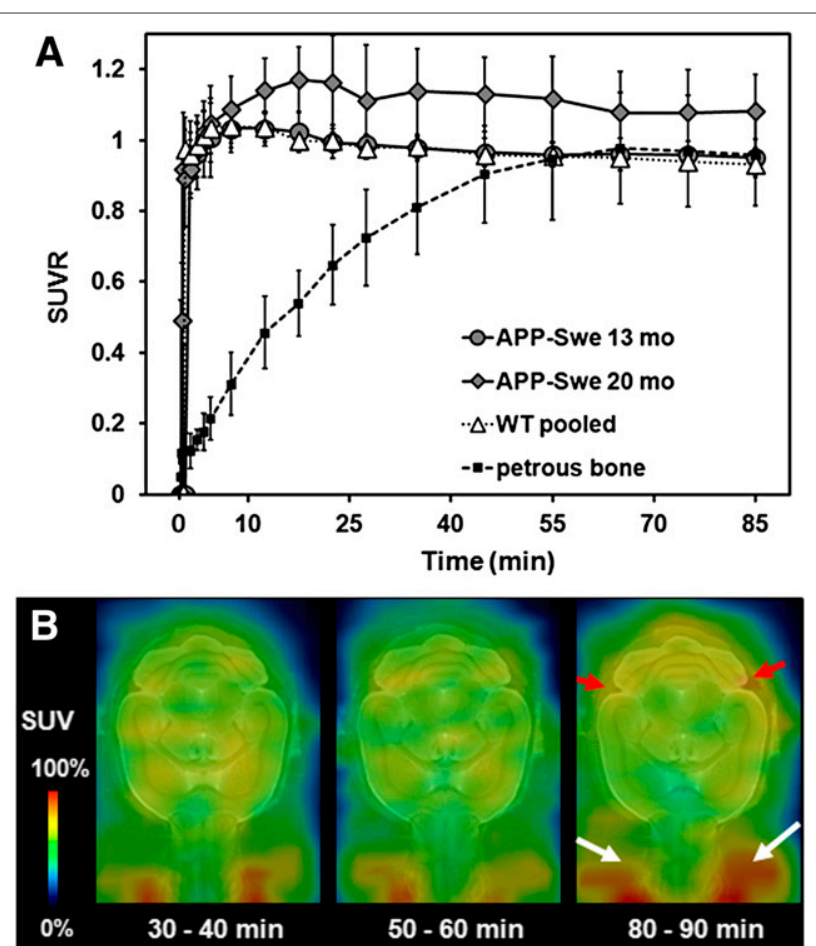

C

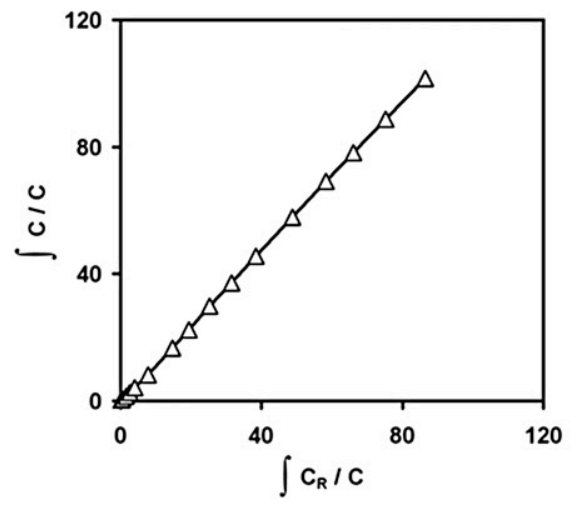

FIGURE 2. (A) Cortex-to-cerebellum ratios and petrous bone-tocerebellum ratio as functions of time after ${ }^{18} \mathrm{~F}$-florbetaben administration in 13- and 20-mo-old transgenic APP-Swe mice and pooled WT mice (10-20 mo). Error bars indicate SD for estimates in groups of 5-10 transgenic and all WT animals. (B) Representative PET recording from various time frames of transgenic 16-mo old animal. Red arrows indicate progressive extracerebral activity in petrous bone, compared with earlier time frames, and white arrows indicate increased signal in frontal cranium and Harderian glands. (C) Corresponding Logan plot of frontal VOI in aged (20 mo) transgenic APP-Swe mice.

activity in petrous bone reached the cerebellar level at times after $60 \mathrm{~min}$.

No significant age-dependent changes in ligand binding could be observed in the pilot study or likewise in the WT animals from the transgenic study. VOI-based analyses of the main study revealed significant SUVR differences, compared with baseline estimates (13 mo), in transgenic animals at the age of 16 mo $(+7.9 \% ; P<0.01)$ and an increase by $16.6 \%$ at the age of $20 \mathrm{mo}(P<0.001)$ (Fig. 4A; Table 1).

Results for individual transgenic mice showed a continuous increase in tracer binding, whereas binding in WT animals remained constant over time (Figs. 4B and 4C). Figure 5 shows 2 representative coronal planes through the ventral frontal cortex of mean Logan $\mathrm{BP}_{\mathrm{ND}}$ maps and SUVR maps in both WT and transgenic animals, revealing the increasing cortical uptake with age in transgenic mice.

\section{Correlation with Histochemistry}

Histochemical analyses revealed fibrillar $\beta$-amyloid plaques predominantly in the frontal cortex, complete absence in the cerebellum, and only a small amount in the hippocampus in some animals. In the 2 mice examined at the age of $13 \mathrm{mo}$, the plaque loads were $0.64 \%$ and $0.06 \%$. In all transgenic mice surviving to 20 mo, the mean plaque load was $4.1 \% \pm 2.8 \%$ (range, $1.0 \%-$ $9.2 \%$ ) in the frontal cortex VOI.

The correlation between percentage plaque load from histochemical analyses and SUVRs from PET recordings in similar volumes for the entire study group is depicted in Figure 6; PET results correlate highly significantly with the histochemical quantitation of plaque deposits $(R=0.95, P<0.001)$. Autoradiographic findings ex vivo in representative young and aged transgenic animals are illustrated in Figure 6, and respective quantitation is presented in Table 1 . The punctate distribution of $\beta$-amyloid labeling in the aged transgenic mouse, compared with findings in the young mouse, notably in the frontal cortex, concurs closely with histochemical findings and matches the anatomic location of increased tracer binding seen in the PET images.

\section{Voxelwise Analyses}

Voxelwise group contrasts between transgenic and WT animals are shown in Figure 7. By this exploratory approach, we were able to discern significant differences in ${ }^{18} \mathrm{~F}$-florbetaben uptake between transgenic and WT animals for the first time at 13 mo of age in a small cluster in the right frontal cortex. The spatial extent of this cluster progressed frontally and also parietally with increasing age, comprising 22,935 voxels at the age of $13 \mathrm{mo}, 142,378$ voxels at the age of $16 \mathrm{mo}$, and finally 278,692 voxels at the age of 20 mo (voxel size, $0.06 \times 0.06 \times 0.06 \mathrm{~mm}$ ). No significant difference was observed subcortically, and white matter labeling remained constant over time. Similar results with identical patterns

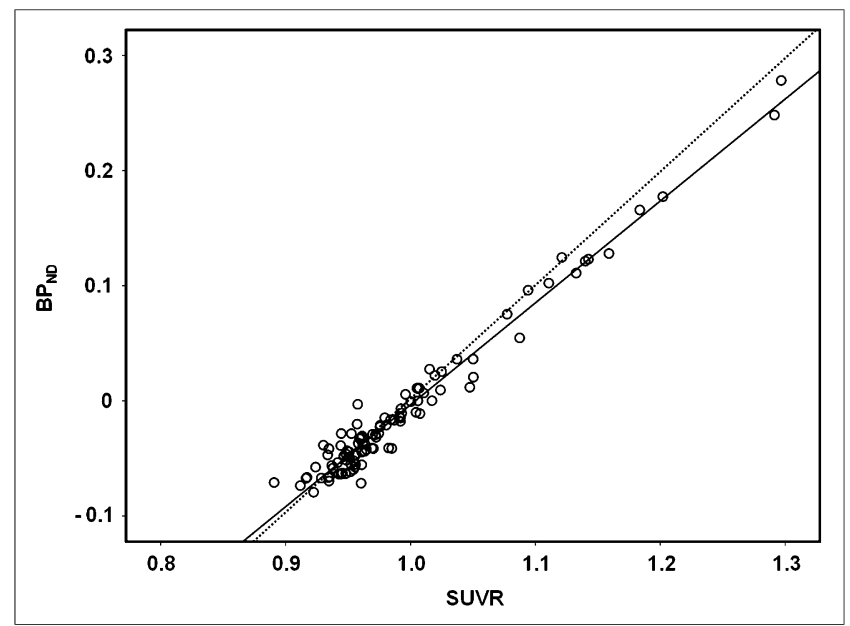

FIGURE 3. Correlation of ${ }^{18} \mathrm{~F}$-florbetaben $\mathrm{BP}_{\mathrm{ND}}$ estimated from 90 -min dynamic PET recordings with SUVR (cortex/cerebellum) results from 30- to 60-min static frame, estimated from cortical VOI in all mice. Dotted line represents perfect line of identity $\left(B P_{N D}=S U V R-1\right)$. 

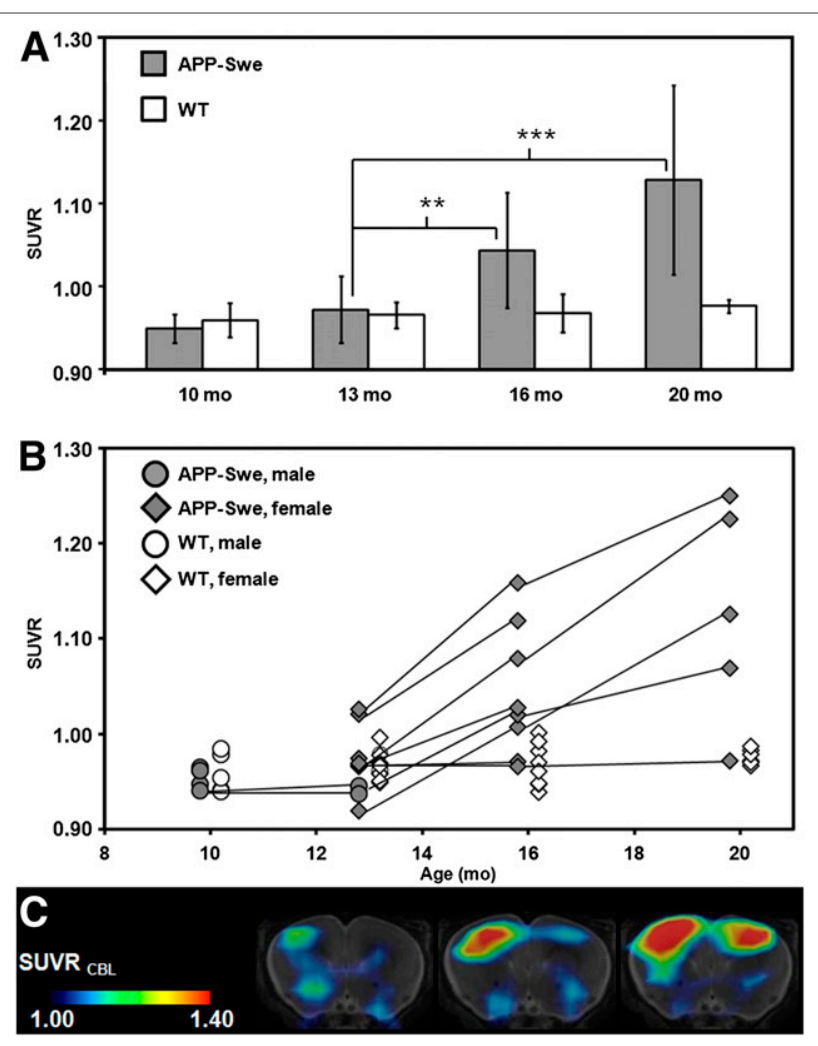

FIGURE 4. (A) VOI-based ${ }^{18} \mathrm{~F}$-florbetaben SUVR (cortex/cerebellum) group comparisons for transgenic APP-Swe mice (gray bar) and WT animals (white bar) aged 10, 13, 16, and 20 mo (mean \pm SD). ${ }^{\star \star} P<0.01$. ${ }^{* \star *} P<0.001$ (2-sample $t$ test). (B) Longitudinal intraindividual follow-up for all mice including pilot and main study animals. Black lines link consecutive data points for single transgenic animals (gray), whereas WT mice are depicted in white. Circles indicate male mice while diamonds display female mice. (C) Representative example for increasing signal over time for single transgenic mouse at the age of 13, 16, and 20 mo (left to right). Coronal planes show SUVR maps through ventral frontal cortex projected on MR imaging mouse atlas (gray scale). Asymmetry (right $>$ left) with $11.4 \%$ at 20 mo of age was observed by small-animal PET and confirmed by histochemical analysis in this animal.

were obtained when contrasted against baseline scans of the transgenic animals. Voxelwise analyses of WT animals did not show any significant change in cortical or subcortical binding over time (data not shown).

\section{Asymmetry}

As an incidental finding arising from our approach of analyzing hemispheres separately, we noted some asymmetry of $\beta$-amyloid deposition in this mouse model. At the age of 20 mo, 2 animals showed a laterality index $((\mathrm{L}-\mathrm{R}) /(\mathrm{L}+\mathrm{R}))(22)$ of greater than -0.04 in the PET scan (Fig. 4C), which was confirmed in the histochemical analyses and also the SPM voxelwise analysis- that is, greater ${ }^{18} \mathrm{~F}$-florbetaben uptake on the right side for the whole group (Fig. 7). This asymmetry tended to progressively develop with age in these mice.

\section{VOI-Based and Histochemical Analyses in Hippocampus}

There were no significant group differences for the hippocampal SUVR in the VOI-based analyses in mice at 20 mo of age, compared with baseline estimates $(1.06 \pm 0.10$ vs. $1.02 \pm 0.05$; $p=$ not significant); histochemical analysis revealed only very few plaques in this area for the group as a whole. In 1 of 7 transgenic mice, there were obviously more hippocampal plaques at the age of 20 mo (1.8\%, compared with $<1 \%)$, which concurred with the individual PET findings of higher SUVR than the mean of the whole transgenic group (1.22 vs. $1.06 \pm 0.10)$.

\section{DISCUSSION}

We have performed the first, to our knowledge, longitudinal PET study in an AD mouse model using the novel amyloid tracer ${ }^{18}$ F-florbetaben. Small-animal PET imaging for the sensitive monitoring of differences between transgenic and WT animals remains a challenge, due especially to the limited spatial resolution relative to the scale of target structures - that is, partial-volume effectsand, in the case of ${ }^{18} \mathrm{~F}$-labeled radiotracers such as ${ }^{18} \mathrm{~F}$-florbetaben, progressively increasing spillover of radioactivity from skull to brain. Despite these potential limitations, our PET procedure for quantitation of $\beta$-amyloid burden proved to be robust, yielding results that concur closely with histochemical findings and ex vivo autoradiography. We find that SUVR estimates derived from static recordings in the interval of 30-60 min after tracer injection give an excellent correlation with steady-state estimates of $\mathrm{BP}_{\mathrm{ND}}$ from 90-min recordings; this approach with truncated acquisitions should reduce the loss of transgenic animals and facilitate scanning of larger groups in planned intervention studies.

The applicability of small-animal PET for monitoring $\beta$-amyloid accumulation has been a matter of some controversy. In 1 study with the bivalent radioligand 2-(1-\{6-[(2-18 F-fluoroethyl)(methyl)amino]2-naphthyl \}ethylidene)malononitrile ( ${ }^{18} \mathrm{~F}$-FDDNP), which has high affinity for $\beta$-amyloid and also neurofibrillary tangles, there was no measurable increase in cerebral binding in 13- to 15 -mo-old $\mathrm{Tg} 2576$ mice (23). This negative result led the authors to conclude that factors related to spatial resolution might fundamentally limit the suitability of molecular imaging for translational AD research in transgenic mice. Indeed, longitudinal PET studies with ${ }^{18} \mathrm{~F}$ FDDNP in triple-transgenic rats show increased binding in the frontal cortex and hippocampus, first evident at the age of $12 \mathrm{mo}$ (24). This discrepancy may well reflect the greater target size in rats and consequently lesser partial-volume effects. Although the

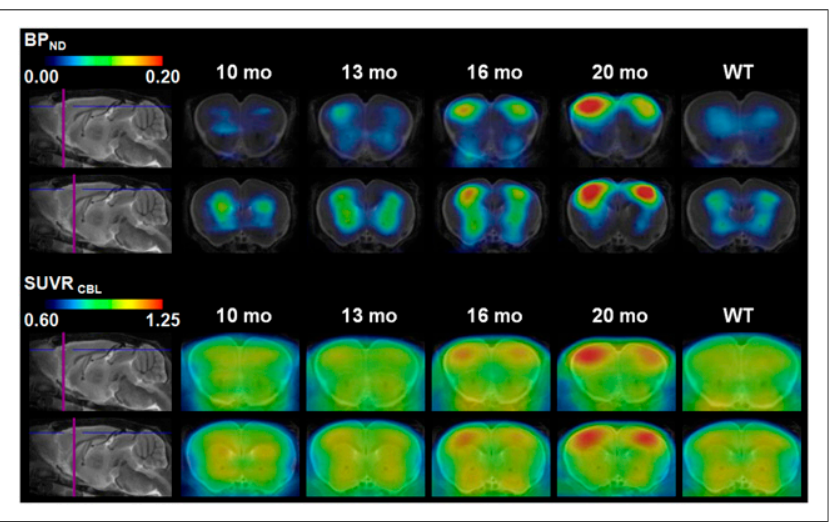

FIGURE 5. Coronal planes of mean ${ }^{18} \mathrm{~F}$-florbetaben $\mathrm{BP}_{\mathrm{ND}}$ maps and corresponding mean SUVR maps through ventral frontal cortex at different ages of transgenic animals $(10,13,16$, and $20 \mathrm{mo})$ and pooled WT animals (10-20 mo; mean, $14.8 \pm 3.4 \mathrm{mo}$ ) projected on MR imaging mouse atlas (gray scale). 


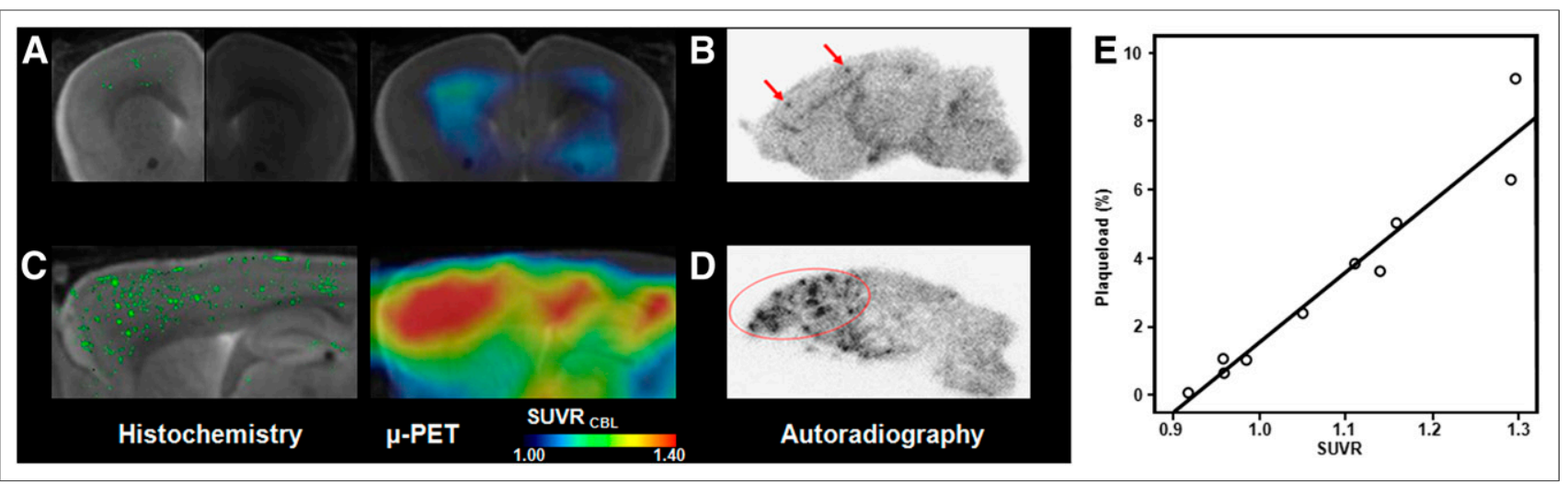

FIGURE 6. Multimodal imaging results for histochemical methoxy-X04 staining along with ${ }^{18} \mathrm{~F}$-florbetaben SUVR maps projected on MR imaging mouse atlas (gray scale) in vivo and corresponding images for autoradiography ex vivo. (A) Histochemistry in right hemisphere (bright side of image) and corresponding small-animal PET shown in coronal slice of 13-mo-old transgenic APP-Swe mouse. (B) Corresponding autoradiography in sagittal plane of left hemisphere in same animal. (C) Histochemistry in right hemisphere and corresponding small-animal PET shown in sagittal slice of 20-mo-old transgenic APP-Swe mouse. (D) Corresponding autoradiography in sagittal plane of left hemisphere in same animal. (E) Correlation of percentage plaque load as determined from histochemistry with SUVR analyses of ${ }^{18} \mathrm{~F}$-florbetaben PET in group of APP-Swe mice aged 13-20 mo. $\mu$-PET = small-animal PET.

comparison of small-animal PET studies are difficult because of the use of different animal models, radiotracers, and study designs, our finding of progressive labeling of $\beta$-amyloid with ${ }^{18} \mathrm{~F}$-florbetaben in the cerebral cortex of APP-Swe mice stands in contrast to the earlier ${ }^{18} \mathrm{~F}$-FDDNP study in $\mathrm{Tg} 2576$ mice. Our results are also in contrast to those of a study with ${ }^{11} \mathrm{C}$-Pittsburgh compound B $\left({ }^{11} \mathrm{C}-\mathrm{PIB}\right)$ in aged $\mathrm{Tg} 2576$ mice, which had lower cortical binding than did WT mice. That paradoxical finding was attributed to confounding effects of cerebral blood flow and the low abundance of ${ }^{11} \mathrm{C}$-PIB binding sites per plaque as expressed in mouse brain (10). However, a subsequent study with ${ }^{11} \mathrm{C}$-PIB of high specific activity $(>200 \mathrm{GBq} / \mu \mathrm{mol})$ showed considerably more uptake in the cerebral cortex of aged APP23 mice than was seen in age-matched healthy controls (7). In that same study, there was an excellent correlation between PET results in the neocortex and those in the archicortex (i.e., hippocampus) and local $\beta$-amyloid burden (percentage) measured by histology —as likewise seen in the present ${ }^{18} \mathrm{~F}$-florbetaben study for the neocortex-but not in the hippocampus, in which specific signal was discernible only in 1 of 5 most aged APP-Swe mice. Thus, the factors accounting for

the regional pattern of $\beta$-amyloid deposition in different transgenic mouse strains remain to be established. However, our findings generally concur with a recent multimodal validation of ${ }^{11} \mathrm{C}$-PIB for PET studies of APP/PS1 mice, which showed excellent correlations between PET findings and the measurements of plaque burden obtained ex vivo and in vitro in the same animals (8), as likewise seen with another high-affinity ${ }^{11} \mathrm{C}$-ligand based on the imidazo[2,1-b]benzothiazole scaffold (25). The manner in which different $\beta$-amyloid isoform patterns and the degree of fibrillarity contribute to PET imaging results remains to be elucidated. In a previous study in which the total amount of $\beta$-amyloid and its isoforms were considered (8), there was a highly significant correlation of total $A \beta_{40}$ and $\mathrm{A} \beta_{42}$ quantification as assessed by enzyme-linked immunosorbent assay and histochemical findings (Thioflavin $\mathrm{S}$ staining) in forebrain structures. Notwithstanding, ${ }^{18}$ F-florbetaben preferably binds to fibrillary plaques rather than diffuse amyloid (14), which is in concordance with the binding characteristics of the methoxy-X04 staining (26) performed in our study.

The authors of positive $\beta$-amyloid PET studies in transgenic mice have generally emphasized the impor-
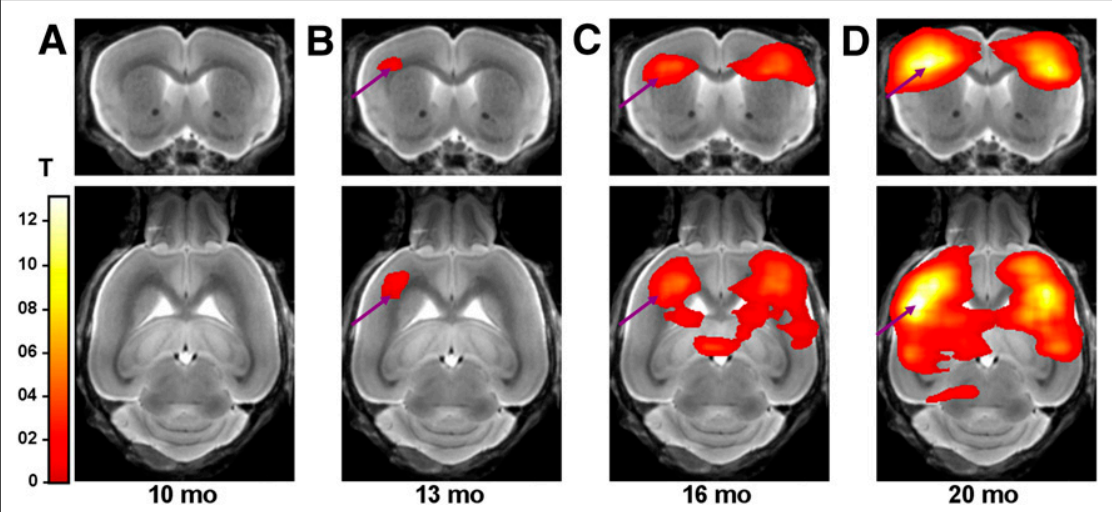

FIGURE 7. Longitudinal voxelwise group comparisons of ${ }^{18} \mathrm{~F}$-florbetaben SUVR maps in transgenic APP-Swe versus pooled WT animals $(n=27$; age, 10-20 mo) with increasing age: $10(\mathrm{~A}), 13(\mathrm{~B}), 16(\mathrm{C})$, and 20 (D) mo. 2-sample $t$ test, $P<0.001$, uncorrected for multiple comparisons, $\mathrm{k}>20$ voxel, projected on MR imaging mouse atlas (gray scale). tance of accurate, quality-controlled manual coregistrations and optimal procedures for image reconstruction, especially in studies of longitudinal design. On the basis of present experience, we concur with this conclusion and note that PET/CT and PET/MR multimodal imaging should facilitate spatial normalization in future studies, but that normalization is by no means absolutely essential.

The physical half-life of ${ }^{18} \mathrm{~F}$, which is 109 min, presents a practical advantage in the design of PET studies with tracers such as ${ }^{18} \mathrm{~F}$-FDDNP and ${ }^{18} \mathrm{~F}$-florbetaben and is furthermore a decisive factor for research centers lacking an on-site cyclotron or radiochemistry facility. However, rodents are especially apt to defluorinate many ${ }^{18} \mathrm{~F}$-labeled 
radiotracers, resulting in progressive labeling of the skeleton; spillover of radioactivity from the cranium to the brain can present a considerable obstacle to quantitation of brain PET, despite optimal image reconstruction with spatial resolution on the order of $1 \mathrm{~mm}$. We have previously reported on the consequences of cranial labeling for quantitation of the cerebral binding of ${ }^{18} \mathrm{~F}$-labeled benzamides to dopamine receptors $(27,28)$. In those studies, progressive contamination of the cerebellum reference tissue signal contributed importantly to a systematic underestimation of the magnitude of the ligand $\mathrm{BP}_{\mathrm{ND}}$ in the striatum region of interest, despite an optimized iterative reconstruction procedure. Others have resorted to factor analysis for the correction of spill-in (29) or pharmacologic suppression of the pathway for radioligand defluorination (30). In the only previous rodent study using the ${ }^{18} \mathrm{~F}$-labeled amyloid tracer flutemetamol considering this issue (9), no significant defluorination was detected in rats in vivo, and the uptake in bone and brain were comparable at $120 \mathrm{~min}$ after injection. Similarly, we find that the magnitude of the cranial signal approaches that of the cerebellum reference tissue during ${ }^{18} \mathrm{~F}$ florbetaben recordings after $60 \mathrm{~min}$ (Figs. 2A and 2B). The timedependent increase in net spillover to cerebral cortex propagates to declining SUVR and $\mathrm{BP}_{\mathrm{ND}}$ estimates for circulation times after $60 \mathrm{~min}$. However, we can substantially avoid this bias by selecting the time window of $30-60 \mathrm{~min}$ for ${ }^{18} \mathrm{~F}$-florbetaben quantitation and note that defluorination of this tracer occurs to a much lesser degree than in benzamide derivatives.

Unspecific white matter binding is a common feature of ${ }^{18} \mathrm{~F}$ labeled amyloid tracers $(31,32)$ and to a lesser degree also of ${ }^{11} \mathrm{C}$ PIB. The cerebral distributions of ${ }^{18} \mathrm{~F}$-flutemetamol and ${ }^{11} \mathrm{C}$-PIB were compared in a recent PET and ex vivo and in vitro autoradiography study of Tg2576 mice and WT animals (9), revealing a slower clearance from white matter of the former compound, in line with its higher lipophilicity. We did not investigate plasma metabolites of the radiotracer in the present mouse study; as described earlier in humans (33), the preponderance of labeled metabolites was more hydrophilic than the parent compound, predicting poor penetration of metabolites into the brain. Retention of ${ }^{18} \mathrm{~F}-$ florbetaben in the white matter of human brain exceeded that of ${ }^{11} \mathrm{C}$-PIB (32). Because present PET methods cannot resolve cortical gray and white matter in mice, we measure in effect weighted mean radioactivity concentrations in the neocortex and in the cerebellum reference tissue. However, preferential binding in white matter can hardly be discerned, even by ex vivo autoradiography (Fig. 6). In any event, white matter contributions will tend to cancel out in SUVR and $\mathrm{BP}_{\mathrm{ND}}$ calculations, a contention supported by the absolute lack of any spurious cortical signal in WT animals and young transgenic animals - that is, SUVR is 1 and $\mathrm{BP}_{\mathrm{ND}}$ is 0 , as likewise reported in earlier reports with ${ }^{11} \mathrm{C}$-PIB $(8,34)$. This lack of bias in baseline estimates of $\beta$-amyloid deposition allows us to estimate the sensitivity of our method for detecting $\beta$-amyloid to be approximately $1.5 \%$ plaque load (derived from Fig. $6 \mathrm{E}$ ) in a defined area, relative to gold standard histology results.

Our voxelwise analysis detected a significant group difference in mice at the age of $13 \mathrm{mo}$, thus proving the suitability for the detection of mild neocortical amyloidogenesis in mice, as is well known in human PET studies of $\mathrm{AD}(35,36)$. In a recent study with the ${ }^{18} \mathrm{~F}$-florbetaben analog ${ }^{18} \mathrm{~F}$-florbetapir $(11)$, increased binding in the cerebral cortex of APP/PS1 mice was already evident at 5 mo of age $(+9 \%$ increase), but there was no evidence for a progressive increase by 12 mo. However, our longitudinal design revealed the spatial progression of cortical $\beta$-amyloid deposition in APP-Swe mice, and the present voxelwise statistical approach was particularly suited for revealing this amyloidogenesis (Fig. 7). This approach should serve in longitudinal intervention studies aimed at attenuating $\beta$-amyloid burden.

Whereas previous PET investigations in other AD models found symmetric labeling (8), a recent gene expression study has shown that transthyretin, a protein sequestering $\beta$-amyloid, was asymmetrically expressed in the adult brain of Tg2576 mice (37). We detected the first evidence of $\beta$-amyloid deposition on the right side of APP-Swe mice aged $13 \mathrm{mo}$, and this asymmetry, although small in magnitude, tended to remain in the subsequent PET investigations. Further investigation in larger groups would be required to determine whether this was a false-positive finding.

It was argued in a previous study using ${ }^{18} \mathrm{~F}$-florbetapir that the spatial resolution of current PET may simply be inadequate to detect $\beta$-amyloid in structures as small as the mouse hippocampus (11), although we note that it is the source intensity rather than spatial extent that ultimately determines the sensitivity of PET. We found the extent of hippocampal amyloidogenesis in APP-Swe mice to be vanishingly low in most mice, as proven by histochemistry. As the only mouse developing manifest $\beta$-amyloid plaques in the hippocampus showed higher plaque load in cortical areas as well, this finding was probably related to the overall penetrance of the trait. Hippocampal $\beta$-amyloid was reliably detected in a ${ }^{11} \mathrm{C}$-PIB study of APP23 mice aged 20 mo (7); at present, it is uncertain if this reflects inherently greater sensitivity of the tracer or more likely the inherently greater penetrance of $\beta$-amyloid accumulation in the particular disease model.

We encountered a relatively high interanimal variability in percentage plaque burden in the transgenic animals, as confirmed by histochemical analyses. Not all animals could be followed to $20 \mathrm{mo}$; a transgenic model with faster plaque accumulation (such as doubleor triple-transgenics) might present advantages arising from better anesthesia tolerance and general health in younger mice. However, because of our finding of adequate quantitation by shorter acquisition times, drop-out rates should decline in future longitudinal studies. Autoradiography was available only in a subset of animals and not in conjunction with histochemistry for the same hemisphere. Specific signal in the hippocampus of APP-Swe mice was too low for the reliable detection of $\beta$-amyloid by ${ }^{18} \mathrm{~F}$-florbetaben PET. Total amounts of soluble and insoluble $\beta$-amyloid species in mouse brain tissues as assessed by sandwich enzyme-linked immunosorbent assay were not performed, but in any event, we emphasize the fibrillary component for cross-validation of the PET signal.

\section{CONCLUSION}

In the most extensive longitudinal PET study yet conducted in an AD mouse model using an ${ }^{18} \mathrm{~F}$-labeled amyloid tracer, we demonstrated the first appearance of discernible $\beta$-amyloid plaque load at 13 mo and a progression to 20 mo. The magnitude of $\mathrm{BP}_{\mathrm{ND}}$ calculated relative to the cerebellum was assessed by $90-\mathrm{min}$ dynamic PET recordings but correlated precisely with SUVR measurements from static imaging at 30-60 min after injection. Furthermore, PET results with ${ }^{18} \mathrm{~F}$-florbetaben correlated closely with gold-standard histochemical analyses. SPM analysis of ${ }^{18} \mathrm{~F}$-florbetaben binding promises to serve admirably for monitoring the effects of interventions in longitudinal studies of APP-Swe mice. 


\section{DISCLOSURE}

The costs of publication of this article were defrayed in part by the payment of page charges. Therefore, and solely to indicate this fact, this article is hereby marked "advertisement" in accordance with 18 USC section 1734 . The study was sponsored by grants from SFB 596. No other potential conflict of interest relevant to this article was reported.

\section{ACKNOWLEDGMENTS}

Florbetaben precursor was kindly provided by Bayer Pharma AG. APP-Swe mice were generously provided by F. Hoffmann-La Roche AG. We thank Heidrun Zankl and Rosel Oos for excellent technical assistance.

\section{REFERENCES}

1. Ziegler-Graham K, Brookmeyer R, Johnson E, Arrighi HM. Worldwide variation in the doubling time of Alzheimer's disease incidence rates. Alzheimers Dement. 2008;4:316-323.

2. Weiner MW, Veitch DP, Aisen PS, et al. The Alzheimer's Disease Neuroimaging Initiative: a review of papers published since its inception. Alzheimers Dement. 2012;8:S1-S68.

3. Duyckaerts C, Delatour B, Potier MC. Classification and basic pathology of Alzheimer disease. Acta Neuropathol. 2009;118:5-36.

4. Hall AM, Roberson ED. Mouse models of Alzheimer's disease. Brain Res Bull. 2012;88:3-12.

5. Teipel SJ, Buchert R, Thome J, Hampel H, Pahnke J. Development of Alzheimerdisease neuroimaging-biomarkers using mouse models with amyloid-precursor protein-transgene expression. Prog Neurobiol. 2011;95:547-556.

6. Jucker M. The benefits and limitations of animal models for translational research in neurodegenerative diseases. Nat Med. 2010;16:1210-1214.

7. Maeda J, Ji B, Irie T, et al. Longitudinal, quantitative assessment of amyloid, neuroinflammation, and anti-amyloid treatment in a living mouse model of Alzheimer's disease enabled by positron emission tomography. J Neurosci. 2007;27:10957-10968.

8. Manook A, Yousefi BH, Willuweit A, et al. Small-animal PET imaging of amyloid-beta plaques with $\left[{ }^{11} \mathrm{C}\right] \mathrm{PiB}$ and its multi-modal validation in an APP/ PS1 mouse model of Alzheimer's disease. PLOS ONE. 2012;7:e31310.

9. Snellman A, Rokka J, Lopez-Picon FR, et al. Pharmacokinetics of $\left[{ }^{18} \mathrm{~F}\right]$ flutemetamol in wild-type rodents and its binding to beta amyloid deposits in a mouse model of Alzheimer's disease. Eur J Nucl Med Mol Imaging. 2012;39:1784-1795.

10. Toyama H, Ye D, Ichise M, et al. PET imaging of brain with the beta-amyloid probe, $\left[{ }^{11} \mathrm{C}\right] 6-\mathrm{OH}-\mathrm{BTA}-1$, in a transgenic mouse model of Alzheimer's disease. Eur J Nucl Med Mol Imaging. 2005;32:593-600.

11. Poisnel G, Dhilly M, Moustie O, et al. PET imaging with $\left[{ }^{18} \mathrm{~F}\right] \mathrm{AV}-45$ in an APP/ PS1-21 murine model of amyloid plaque deposition. Neurobiol Aging. 2012;33:2561-2571.

12. Zhang W, Oya S, Kung MP, Hou C, Maier DL, Kung HF. F-18 polyethyleneglycol stilbenes as PET imaging agents targeting Abeta aggregates in the brain. Nucl Med Biol. 2005;32:799-809.

13. Zhang W, Oya S, Kung MP, Hou C, Maier DL, Kung HF. F-18 stilbenes as PET imaging agents for detecting beta-amyloid plaques in the brain. J Med Chem. 2005;48:5980-5988.

14. Fodero-Tavoletti MT, Brockschnieder D, Villemagne VL, et al. In vitro characterization of $\left[{ }^{18} \mathrm{~F}\right]$-florbetaben, an Abeta imaging radiotracer. $\mathrm{Nucl} \mathrm{Med} \mathrm{Biol.}$ 2012;39:1042-1048.

15. Barthel H, Gertz HJ, Dresel S, et al. Cerebral amyloid-beta PET with florbetaben $\left({ }^{18} \mathrm{~F}\right)$ in patients with Alzheimer's disease and healthy controls: a multicentre phase 2 diagnostic study. Lancet Neurol. 2011;10:424-435.

16. Richards JG, Higgins GA, Ouagazzal AM, et al. PS2APP transgenic mice, coexpressing hPS2mut and hAPPswe, show age-related cognitive deficits associated with discrete brain amyloid deposition and inflammation. J Neurosci. 2003;23:8989-9003.
17. Rominger A, Mille E, Zhang S, et al. Validation of the octamouse for simultaneous ${ }^{18} \mathrm{~F}$-fallypride small-animal PET recordings from 8 mice. $\mathrm{J} \mathrm{Nucl} \mathrm{Med.}$ 2010;51:1576-1583.

18. Dorr A, Sled JG, Kabani N. Three-dimensional cerebral vasculature of the CBA mouse brain: a magnetic resonance imaging and micro computed tomography study. Neuroimage. 2007;35:1409-1423.

19. Constantinescu CC, Mukherjee J. Performance evaluation of an Inveon PET preclinical scanner. Phys Med Biol. 2009;54:2885-2899.

20. Logan J, Fowler JS, Volkow ND, Wang GJ, Ding YS, Alexoff DL. Distribution volume ratios without blood sampling from graphical analysis of PET data. J Cereb Blood Flow Metab. 1996;16:834-840.

21. Sawiak SJ, Wood NI, Williams GB, Morton AJ, Carpenter TA. Voxel-based morphometry in the R6/2 transgenic mouse reveals differences between genotypes not seen with manual 2D morphometry. Neurobiol Dis. 2009;33: 20-27.

22. Vernaleken I, Weibrich C, Siessmeier T, et al. Asymmetry in dopamine $D(2 / 3)$ receptors of caudate nucleus is lost with age. Neuroimage. 2007;34:870-878.

23. Kuntner C, Kesner AL, Bauer M, et al. Limitations of small animal PET imaging with $\left[{ }^{18} \mathrm{~F}\right] \mathrm{FDDNP}$ and FDG for quantitative studies in a transgenic mouse model of Alzheimer's disease. Mol Imaging Biol. 2009;11:236-240.

24. Teng E, Kepe V, Frautschy SA, et al. [F-18]FDDNP microPET imaging correlates with brain Abeta burden in a transgenic rat model of Alzheimer disease: effects of aging, in vivo blockade, and anti-Abeta antibody treatment. Neurobiol Dis. 2011;43:565-575.

25. Yousefi BH, Manook A, Drzezga A, et al. Synthesis and evaluation of ${ }^{11} \mathrm{C}$ labeled imidazo[2,1-b]benzothiazoles (IBTs) as PET tracers for imaging betaamyloid plaques in Alzheimer's disease. J Med Chem. 2011;54:949-956.

26. Klunk WE, Bacskai BJ, Mathis CA, et al. Imaging Abeta plaques in living transgenic mice with multiphoton microscopy and methoxy-X04, a systemically administered Congo red derivative. J Neuropathol Exp Neurol. 2002;61:797-805.

27. Rominger A, Wagner E, Mille E, et al. Endogenous competition against binding of $\left[{ }^{18} \mathrm{~F}\right] \mathrm{DMFP}$ and $\left[{ }^{18} \mathrm{~F}\right]$ fallypride to dopamine $\mathrm{D}_{2 / 3}$ receptors in brain of living mouse. Synapse. 2010;64:313-322.

28. Mille E, Cumming P, Rominger A, et al. Compensation for cranial spill-in into the cerebellum improves quantitation of striatal dopamine $D_{2 / 3}$ receptors in rats with prolonged $\left[{ }^{18} \mathrm{~F}\right]$-DMFP infusions. Synapse. 2012;66:705-713.

29. Millet P, Moulin-Sallanon M, Tournier BB, et al. Quantification of dopamine $\mathrm{D}_{2 / 3}$ receptors in rat brain using factor analysis corrected $\left[{ }^{18} \mathrm{~F}\right]$ fallypride images. Neuroimage. 2012;62:1455-1468.

30. Choi SR, Schneider JA, Bennett DA, et al. Correlation of amyloid PET ligand florbetapir F 18 binding with Abeta aggregation and neuritic plaque deposition in postmortem brain tissue. Alzheimer Dis Assoc Disord. 2012;26:8-16.

31. Herholz K, Ebmeier K. Clinical amyloid imaging in Alzheimer's disease. Lancet Neurol. 2011;10:667-670.

32. Rowe CC, Villemagne VL. Brain amyloid imaging. J Nucl Med. 2011;52:17331740 .

33. Patt M, Schildan A, Barthel H, et al. Metabolite analysis of $\left[{ }^{18} \mathrm{~F}\right]$ Florbetaben (BAY 94-9172) in human subjects: a substudy within a proof of mechanism clinical trial. J Radioanal Nucl Chem. 2010;284:557-562.

34. Maeda J, Zhang MR, Okauchi T, et al. In vivo positron emission tomographic imaging of glial responses to amyloid-beta and tau pathologies in mouse models of Alzheimer's disease and related disorders. J Neurosci. 2011;31: 4720-4730.

35. Barthel $\mathrm{H}$, Luthardt $\mathrm{J}$, Becker $\mathrm{G}$, et al. Individualized quantification of brain beta-amyloid burden: results of a proof of mechanism phase 0 florbetaben PET trial in patients with Alzheimer's disease and healthy controls. Eur J Nucl Med Mol Imaging. 2011;38:1702-1714.

36. Förster S, Grimmer T, Miederer I, et al. Regional expansion of hypometabolism in Alzheimer's disease follows amyloid deposition with temporal delay. Biol Psychiatry. 2012;71:792-797.

37. Tsai KJ, Yang CH, Lee PC, Wang WT, Chiu MJ, Shen CK. Asymmetric expression patterns of brain transthyretin in normal mice and a transgenic mouse model of Alzheimer's disease. Neuroscience. 2009;159:638-646. 